\title{
MOUNTAIN CLIMBING
}

\author{
BY \\ JOHN PHILIP HUNEKE
}

Let "function" mean "continuous function mapping the closed unit interval onto itself"; let $f$ and $g$ be two arbitrary functions. The object of this paper is to determine conditions for the existence of functions $h$ and $j$ such that $f j=g h$ (i.e. $f(j(x))=g(h(x))$ for all $x$ in $[0,1])$.

The existence of such functions became important [1] in constructing commuting functions with appropriate properties. Considering the graphs of $f$ and $g$ to be mountains (as in [2]) $h$ and $j$ denote the horizontal progress of climbers, one on each mountain, who are trying to maintain the same elevation as each other (time represented by the domain of $h$ and $j$ ).

The following example shows that functions $h$ and $j$ do not always exist (and hence climbers cannot always climb mountains maintaining a common elevation). Define functions $\bar{f}$ and $\bar{g}$ as follows:

$$
\begin{aligned}
& \bar{f}(x)=\frac{1}{2}-\left(\frac{1}{2}-x\right) \cdot \cos \left(\pi /\left(\frac{1}{2}-x\right)\right) \text { for } x \text { in }\left[0, \frac{1}{2}\right] ; \\
& \bar{f}(x)=x \text { for } x \text { in }\left[\frac{1}{2}, 1\right] ; \\
& \bar{g}(x)=2 x \text { for } x \text { in }\left[0, \frac{1}{4}\right] ; \\
& \bar{g}(x)=\frac{1}{2} \text { for } x \text { in }\left[\frac{1}{4}, \frac{1}{2}\right] ; \text { and } \\
& \bar{g}(x)=x \text { for } x \text { in }\left[\frac{1}{2}, 1\right] .
\end{aligned}
$$

Any functions $\bar{h}$ and $j$ with the property $\bar{f} \bar{j}=\bar{g} h$ must clearly have $j^{-1}(1)$ $=\bar{h}^{-1}(1)$ and $j^{-1}(0)=\bar{h}^{-1}(0)$, so $\bar{j}^{-1}\left(\frac{1}{2}\right)=\bar{h}^{-1}\left(\frac{1}{2}\right)$. Therefore $\bar{h}^{-1}\left(\left[\frac{1}{4}, \frac{1}{2}\right]\right)$ must have an infinite number of components. These components must have a cluster point $x$, and $x$ is a cluster point for $\bar{h}^{-1}\left(\frac{1}{4}\right)$ and for $\bar{h}^{-1}\left(\frac{1}{2}\right)$. Therefore $\bar{h}$ must not be continuous.

The aim of this paper is to show that the above example furnishes essentially the only condition which could keep the desired functions ( $h$ and $j$ ) from existing, and hence there is always a way for the climbers to climb their respective mountains, maintaining almost the same elevation (i.e. the same elevation except for some arbitrarily small error), provided they start at the lowest point together and seek summits which have the same elevation. In the notation for an interval, $[a, b]$ does not imply $a<b$; i.e. $[a, b]=[b, a]$.

DEFINITIONS 1 AND 2. When $f([a, b])=g([c, d])$, the finite chain of quadruples $P=\left\{p_{i} / i=0,1, \ldots, n\right\}$, where $p_{i}=\left\langle w_{i}, x_{i}, y_{i}, z_{i}\right\rangle$ and where $P$ has order induced by the indices $\left(p_{i}<p_{i^{\prime}}\right.$ if and only if $\left.i<i^{\prime}\right)$, will be called an $\left.f\right|_{[a, b]}-\left.g\right|_{[c, d]}$ weakly compatible chain provided:

Presented to the Society, March 1, 1968; received by the editors March 1, 1968 and, in revised form, June 17, 1968. 
$\{a, b\} \subset\left\{w_{i} / i=0,1, \ldots, n\right\} \cup\left\{x_{i} / i=0,1, \ldots, n\right\} \subset[a, b] ;$

$\{c, d\} \subset\left\{y_{i} / i=0,1, \ldots, n\right\} \cup\left\{z_{i} / i=0,1, \ldots, n\right\} \subset[c, d] ;$

$\left[w_{i}, x_{i}\right] \cap\left[w_{i-1}, x_{i-1}\right]$ is not empty for $i=1, \ldots, n$;

$\left[y_{i}, z_{i}\right] \cap\left[y_{i-1}, z_{i-1}\right]$ is not empty for $i=1, \ldots, n$; and

$f\left(\left[w_{i}, x_{i}\right]\right)=g\left(\left[y_{i}, z_{i}\right]\right)$ for $i=0,1, \ldots, n$.

Also, $P$ will be called an $\left.f\right|_{[a, b]}-\left.g\right|_{[c, d]}$ strongly compatible chain provided: $P$ is an $\left.f\right|_{[a, b]}-\left.g\right|_{[c, d]}$ weakly compatible chain; $x_{i-1}=w_{i}$ and $z_{i-1}=y_{i}$ for each $i=1, \ldots, n$; $f\left(w_{0}\right)=g\left(y_{0}\right)$; and, the four intervals $f\left(\left[w_{i}, x_{i}\right]\right), g\left(\left[y_{i}, z_{i}\right]\right),\left[f\left(w_{i}\right), f\left(x_{i}\right)\right]$, and $\left[g\left(y_{i}\right), g\left(z_{i}\right)\right]$ coincide for each $i=0, \ldots, n$. Observe that an $\left.f\right|_{[a, b]}-\left.g\right|_{[c, d]}$ strongly compatible chain also could have been described as a finite chain of pairs

$$
\left\{\left\langle w_{i}, y_{i}\right\rangle / i=0,1, \ldots, n+1\right\}
$$

(where $w_{n+1}=x_{n}$ and $y_{n+1}=z_{n}$ above) such that:

$\{a, b\} \subset\left\{w_{i} / i=0,1, \ldots, n+1\right\} \subset[a, b]$;

$\{c, d\} \subset\left\{y_{i} / i=0,1, \ldots, n+1\right\} \subset[c, d]$

$f\left(w_{0}\right)=g\left(y_{0}\right) ;$ and,

the four intervals $f\left(\left[w_{i}, w_{i-1}\right]\right), g\left(\left[y_{i}, y_{i-1}\right]\right),\left[f\left(w_{i}\right), f\left(w_{i-1}\right)\right]$, and $\left[g\left(y_{i}\right), g\left(y_{i-1}\right)\right]$ coincide for each $i=1, \ldots, n+1$.

Partially order the collection of all $f-g$ weakly (respectively strongly) compatible chains as follows. If $P$ and $Q$ are two $f-g$ weakly (resp. strongly) compatible chains, let $P \leqq Q$ provided $P=\left\{p_{i} / i=0,1, \ldots, n\right\}$ (as denoted above) and

$$
Q=\left\{q_{i}^{i} / i^{\prime}=0,1, \ldots, n_{i} ; i=0,1, \ldots, n\right\}
$$

(ordered: $q_{i^{\prime}}^{i}<q_{i^{\prime \prime \prime}}^{i^{\prime \prime}}$ provided $i \leqq i^{\prime \prime}$ and if $i=i^{\prime \prime}$ then $i^{\prime}<i^{\prime \prime \prime}$ ) and for each $i=0,1, \ldots, n$, the subchain of $Q,\left\{q_{i^{i}}^{i} / i^{\prime}=0,1, \ldots, n_{i}\right\}$, is an $\left.f\right|_{\left[w_{l}, x_{i}\right]}-\left.g\right|_{\left[y_{i}, z_{i}\right]}$ weakly (resp. strongly) compatible chain.

For any function $h$ and any interval $[a, b],\left.h\right|_{[a, b]}$ denotes the restriction of $h$ to $[a, b]$; hence $h=\left.h\right|_{[0,1]}$.

Proposition 1. There exist functions $h$ and $j$ such that $f j=g h$ if and only if there exists a linearly ordered set $\left\{P_{i} / i=0,1, \ldots\right\}$ (order defined above and denoted by the indices) of $f-g$ weakly compatible chains such that

$$
\lim _{i \rightarrow \infty} \sup _{i^{\prime}}\left(\left|w_{i^{\prime}}^{i}-x_{i^{\prime}}^{i}\right|+\left|y_{i^{\prime}}^{i}-z_{i^{\prime}}^{i}\right|\right)=0
$$

where $P_{i}=\left\{\left\langle w_{i^{\prime}}^{i}, x_{i^{\prime}}^{i}, y_{i^{\prime}}^{i}, z_{i^{\prime}}^{i}\right\rangle / i^{\prime}=1, \ldots, n_{i}\right\}$ for each $i=0,1, \ldots, n$.

Proof. Let $\left\{P_{i} / i=0,1, \ldots\right\}$ be the set satisfying the properties listed in the proposition; the functions $h$ and $j$ will be constructed. Define two sequences of nested sets $\left\{U_{i} / i=0,1, \ldots\right\}$ and $\left\{V_{i} / i=0,1, \ldots\right\}$ inductively. Let

and let

$$
U_{0}=\bigcup_{i^{\prime}=1}^{n_{*}}\left[w_{i^{\prime}}^{*}, x_{i^{\prime}}^{*}\right] \times\left[\frac{i^{\prime}-1}{n_{*}}, \frac{i^{\prime}}{n_{*}}\right]
$$

$$
V_{0}=\bigcup_{i^{\prime}=1}^{n_{*}}\left[y_{i^{\prime}}^{*}, z_{i^{\prime}}^{*}\right] \times\left[\frac{i^{\prime}-1}{n_{*}}, \frac{i^{\prime}}{n_{*}}\right]
$$


where $P_{0}=\left\{\left\langle w_{i^{\prime}}^{*}, x_{i^{\prime}}^{*}, y_{i^{\prime}}^{*}, z_{i^{\prime}}^{*}\right\rangle \mid i^{\prime}=1, \ldots, n_{*}\right\}$ ordered by the indices. Let $k$ be any nonnegative integer, denote $P_{k}$ by $\left\{\left\langle w_{i^{\prime}}, x_{i^{\prime}}, y_{i^{\prime}}, z_{i^{\prime}}\right\rangle \mid i^{\prime}=1, \ldots, n\right\}$ (ordered by the indices), and suppose $U_{k}$ and $V_{k}$ have been defined having the form:

$$
U_{k}=\bigcup_{i^{\prime}=1}^{n}\left[w_{i^{\prime}}, x_{i^{\prime}}\right] \times\left[a_{i^{\prime}-1}, a_{i^{\prime}}\right]
$$

and

$$
V_{k}=\bigcup_{i^{\prime}=1}^{n}\left[y_{i^{\prime}}, z_{i^{\prime}}\right] \times\left[a_{i^{\prime}-1}, a_{i^{\prime}}\right]
$$

where $\left\{a_{i^{\prime}} / i^{\prime}=0,1, \ldots, n\right\}$ is a strictly increasing sequence of numbers such that $a_{0}=0$ and $a_{n}=1$. Since $\left\{P_{i} / i=0,1, \ldots\right\}$ is a linearly ordered set of $f-g$ weakly compatible chains, $P_{k}<P_{k+1}$, so $P_{k+1}$ can be written as:

$$
P_{k+1}=\left\{\left\langle w_{i^{\prime}}^{i}, x_{i^{i}}^{i}, y_{i^{\prime}}^{i}, z_{i^{\prime}}^{i}\right\rangle / i^{\prime}=1, \ldots, n_{i} ; i=1, \ldots, n\right\}
$$

(ordered primarily by the superindices and secondarily by the subindices) such that for each $i=1, \ldots, n$ the subchain of $P_{k+1},\left\{\left\langle w_{i^{i}}^{i}, x_{i^{\prime}}^{i}, y_{i^{\prime}}^{i}, z_{i^{i}}^{i}\right\rangle \mid i^{\prime}=1, \ldots, n_{i}\right\}$, is an $\left.f\right|_{\left[w_{i}, x_{i}\right]}-\left.g\right|_{\left[y_{i}, z_{i}\right]}$ weakly compatible chain. Define $U_{k+1}$ and $V_{k+1}$ as follows: for each $i=1, \ldots, n$, let $\left\{a_{i^{i}}^{i} / i^{\prime}=0,1, \ldots, n_{i}\right\}$ be a strictly increasing sequence of numbers such that $a_{0}^{i}=a_{i-1}$ and $a_{n_{i}}^{i}=a_{i}$; define

$$
U_{k+1}=\bigcup_{i=1}^{n} \bigcup_{i^{\prime}=1}^{n_{1}}\left[w_{i^{\prime}}^{i}, x_{i^{\prime}}^{i}\right] \times\left[a_{i^{\prime}-1}^{i}, a_{i^{\prime}}^{i^{\prime}}\right]
$$

and

$$
V_{k+1}=\bigcup_{i=1}^{n} \bigcup_{i^{\prime}=1}^{n_{1}}\left[y_{i^{\prime}}^{i}, z_{i^{\prime}}^{i}\right] \times\left[a_{i^{\prime}-1}^{i}, a_{i^{\prime}}^{i}\right] .
$$

For each $i=0,1, \ldots, U_{i}$ and $V_{i}$ are closed connected subsets of $[0,1] \times[0,1]$ which touch each side of the box $[0,1] \times[0,1]$, and $U_{i} \supset U_{i+1}$ and $V_{i} \supset V_{i+1}$. Therefore $\bigcap_{i=0,1}, \ldots U_{i}$ and $\bigcap_{i=0,1, \ldots} V_{i}$ are closed sets which are graphs of functions. Let $j$ be the function whose graph is $\bigcap_{i=0,1, \ldots} U_{i}$ and let $h$ be the function whose graph is $\bigcap_{i=0,1}, \ldots V_{i}$. Considered as the composition of relations, $f U_{i}=g V_{i}$ for each $i=0,1, \ldots$; therefore $f j=g h$, so the desired functions have been constructed.

To prove the converse, let $h$ and $j$ be functions such that $f j=g h$. For each $i=0,1, \ldots$, let

$$
Q_{i}=\left\{\left\langle w_{i^{\prime}}^{i}, x_{i^{\prime}}^{i}, y_{i^{\prime}}^{i}, z_{i^{\prime}}^{i}\right\rangle / i^{\prime}=1, \ldots, 2^{i}\right\}
$$

(ordered by the subindices) be defined:

$$
w_{i^{\prime}}^{i}=\inf j\left(\left[\frac{i^{\prime}-1}{2^{i}}, \frac{i^{\prime}}{2^{i}}\right]\right), \quad x_{i^{\prime}}^{i}=\sup j\left(\left[\frac{i^{\prime}-1}{2^{i}}, \frac{i^{\prime}}{2^{i}}\right]\right), \quad y_{i^{\prime}}^{i}=\inf h\left(\left[\frac{i^{\prime}-1}{2^{i}}, \frac{i^{\prime}}{2^{i}}\right]\right),
$$

and

$$
z_{i^{\prime}}^{i}=\sup h\left(\left[\frac{i^{\prime}-1}{2^{i}}, \frac{i^{\prime}}{2^{i}}\right]\right) \text { for each } i^{\prime}=1, \ldots, 2^{i}
$$


Now $\left\{Q_{i} / i=0,1, \ldots\right\}$ is a linearly ordered set of $f-g$ weakly compatible chains. Also, $\lim _{i \rightarrow \infty} \sup _{i^{\prime}}\left|w_{i^{\prime}}^{i}-x_{i^{\prime}}^{i}\right|=0$ and $\lim _{i \rightarrow \infty} \sup _{i^{\prime}}\left|y_{i^{\prime}}^{i}-z_{i^{\prime}}^{i}\right|=0$. Therefore the proposition is satisfied.

Definition 3. Let $h$ be any function and let $a, b, x$, and $y$ be in $[0,1]$ with $a \leqq b$. Then $\left.h\right|_{[a, b]}$ crosses $y$ at $x$ provided there exists $\delta \geqq 0$ such that $x-\delta$ and $x+\delta$ are in $[0,1] ;\left.h\right|_{[a, b] \cap[x-\delta, x]}$ and $\left.h\right|_{[a, b] \cap[x, x+\delta]}$ are constant; and for every $\varepsilon>0, y$ is in the interior of $h([a, b] \cap[x-\delta-\varepsilon, x+\delta+\varepsilon])$, either $x-\delta=a$ or $\left.h\right|_{[a, b] \cap[x-\delta-\varepsilon, x]}$ is not constant, and either $x+\delta=b$ or $\left.h\right|_{[a, b] \cap[x, x+\delta+\varepsilon]}$ is not constant.

The set of all $x$ such that $\left.h\right|_{[a, b]}$ crosses $y$ at $x$ will be denoted by $\left.h\right|_{[a, b]} ^{c}(y)$. Observe that for each $y$ in $[0,1],\left.h\right|_{[a, b]} ^{c}(y)$ is a subset of $\left.h\right|_{[a, b]} ^{-1}(y)$; also $\left.h\right|_{[a, b]} ^{c}(y)$ is empty if and only if $y$ is not in the interior of $h([a, b])$.

The following constructive Definitions 4 and 5 will be used in Lemma 1 to inductively construct a linearly ordered set of $f-g$ strongly compatible chains which will yield functions $j$ and $h$ as in Proposition 1 .

Definition 4. Let $f$ and $g$ be nowhere locally constant, and let $a, a^{\prime}, b$, and $b^{\prime}$ be in $[0,1]$ such that the four intervals $f\left(\left[a, a^{\prime}\right]\right),\left[f(a), f\left(a^{\prime}\right)\right], g\left(\left[b, b^{\prime}\right]\right)$, and $\left[g(b), g\left(b^{\prime}\right)\right]$ coincide, with $f(a)=g(b)$. The $f-g$ refinement of $\left\langle a, a^{\prime}, b, b^{\prime}\right\rangle$ is the $\left.f\right|_{\left[a, a^{\prime}\right]}-\left.g\right|_{\left[b, b^{\prime}\right]}$ strongly compatible chain

$$
\left\{\left\langle a, a_{1}, b, b_{1}\right\rangle \leqq\left\langle a_{1}, a_{2}, b_{1}, b_{2}\right\rangle \leqq\left\langle a_{2}, a^{\prime}, b_{2}, b^{\prime}\right\rangle\right\}
$$

defined as follows. Let $f\left(\left[a,\left(a+a^{\prime}\right) / 2\right]\right)=\left[f(a), c_{1}\right]$ define $c_{1}$, and pick $d_{1}$ in

$$
\left[a,\left(a+a^{\prime}\right) / 2\right] \cap f^{-1}\left(c_{1}\right)
$$

let $f\left(\left[d_{1}, a^{\prime}\right]\right)=\left[c_{2}, f\left(a^{\prime}\right)\right]$ define $c_{2}$, and pick $d_{2}$ in $\left[d_{1}, a^{\prime}\right] \cap f^{-1}\left(c_{2}\right)$; and let $f\left(\left[a, d_{2}\right]\right)$ $=\left[f(a), c_{3}\right]$ define $c_{3}$, and pick $d_{3}$ in $\left[a, d_{2}\right] \cap f^{-1}\left(c_{3}\right)$.

Case 1. If $c_{2}=f(a)$ or $c_{3}=f\left(a^{\prime}\right)$, then define $a_{1}=d_{3}, a_{2}=d_{2}, b_{1}$ to be the element in $\left[b, b^{\prime}\right] \cap g^{-1}\left(c_{3}\right)$ such that $\left[b, b_{1}\right] \cap g^{-1}\left(c_{3}\right)=\left\{b_{1}\right\}$, and $b_{2}$ to be the element in $\left[b, b_{1}\right] \cap g^{-1}\left(c_{2}\right)$ such that $\left[b_{2}, b_{1}\right] \cap g^{-1}\left(c_{2}\right)=\left\{b_{2}\right\}$.

Case 2. Assume that $c_{2} \neq f(a)$ and $c_{3} \neq f\left(a^{\prime}\right)$. Let $r_{0}$ be the element in $\left[b, b^{\prime}\right]$ $\cap g^{c}\left(c_{3}\right)$ such that $\left.g\right|_{\left[b, r_{0}\right]} ^{c}\left(c_{3}\right)$ is an empty set.

Case 2.1. If $\left.g\right|_{\left[r_{0}, b^{\prime}\right]} ^{c}\left(c_{2}\right)$ is an empty set, then define $a_{1}=d_{3}, a_{2}=d_{2}, b_{1}=r_{0}$, and $b_{2}$ to be the element in $\left[b, b_{1}\right] \cap g^{-1}\left(c_{2}\right)$ such that $\left[b_{2}, b_{1}\right] \cap g^{-1}\left(c_{2}\right)=\left\{b_{2}\right\}$.

Case 2.2. If $\left.g\right|_{\left[r_{0}, b^{\prime}\right]} ^{c}\left(c_{2}\right)$ is not empty, pick $r_{1}$ in $\left.g\right|_{\left[r_{0}, b^{\prime}\right]} ^{c}\left(c_{2}\right)$. Let $g\left(\left[b, r_{1}\right]\right)=\left[g(b), s_{2}\right]$ define $s_{2}$, and pick $r_{2}$ in $\left[b, r_{1}\right] \cap g^{-1}\left(s_{2}\right)$; also let $g\left(\left[r_{2}, b^{\prime}\right]\right)=\left[s_{3}, g\left(b^{\prime}\right)\right]$ define $s_{3}$, and pick $r_{3}$ in $\left[r_{2}, b^{\prime}\right] \cap g^{-1}\left(s_{3}\right)$. Define $a_{1}$ to be the element in $\left[a, a^{\prime}\right] \cap f^{-1}\left(s_{2}\right)$ such that $\left[a, a_{1}\right] \cap f^{-1}\left(s_{2}\right)=\left\{a_{1}\right\}, a_{2}$ to be the element in $\left[a, a_{1}\right] \cap f^{-1}\left(s_{3}\right)$ such that $\left[a_{2}, a_{1}\right] \cap f^{-1}\left(s_{3}\right)=\left\{a_{2}\right\}, b_{1}=r_{3}$ and $b_{2}=r_{2}$.

Definition 5. Let $f$ and $g$ be nowhere constant functions and let $P=\left\{p_{i} / i=0\right.$, $1, \ldots, n\}$ be an $f-g$ strongly compatible chain (ordered by the indices). The $f-g$ refinement of $P$ is the $f-g$ strongly compatible chain $Q=\left\{q_{i}^{i} / i^{\prime}=0,1,2 ; i=0,1, \ldots, n\right\}$ (ordered primarily by the superindices and secondarily by the subindices) such that 
$P \leqq Q$ and for each $i=0,1, \ldots, n$, the subchain $\left\{q_{i^{\prime}}^{i} \mid i^{\prime}=0,1,2\right\}$ of $Q$ is the $f-g$ refinement of $p_{i}$.

LEMMA 1. If $f$ and $g$ are nowhere constant and have fixed points 0 and 1 , then there exist functions $h$ and $j$ such that

$f j=g h$;

$h$ and $j$ are nowhere constant;

$h$ and $j$ have fixed points 0 and 1 ;

if $f^{c}(g(x))$ is a finite set then $h^{c}(x)$ is a finite set; and

if $g^{c}(f(y))$ is a finite set then $j^{c}(y)$ is a finite set.

Proof. Assume that $f$ and $g$ are nowhere constant and have fixed points 0 and 1 . The existence of the desired functions $h$ and $j$ will be demonstrated in several steps, the properties which $h$ and $j$ are to satisfy at each step summarized in parentheses.

Step 1. ( $h$ and $j$ have fixed points 0 and 1 such that $f j=g h$, if $r$ is not in $g^{c} g(r)$ then $h^{c}(r)$ is a finite set, and if $s$ is not in $f^{c} f(s)$ then $j^{c}(s)$ is a finite set.) Define a linearly ordered set of $f-g$ strongly compatible chains $\left\{P_{i} / i=0,1, \ldots\right\}$ inductively on the index $i$. Let $P_{0}=\{\langle 0,1,0,1\rangle\}$. For each positive integer $k$, let $P_{k}$ be the $f-g$ refinement of $P_{k-1}$. Now $\left\{P_{i} / i=0,1, \ldots\right\}$ is a linearly ordered set of $f-g$ strongly compatible chains which satisfy the conditions of the preceding proposition (since $f$ and $g$ are nowhere constant and continuous). Hence functions $h$ and $j$ can be constructed (as in the proof of the preceding proposition) such that $f j=g h$. Also, since the initial element of $P_{i}$ has the form $\langle 0, x, 0, z\rangle$ and the terminal element of $P_{i}$ has the form $\langle w, 1, y, 1\rangle$ for each $i=0,1, \ldots(w, x, y$, and $z$ being points depending on $i$ ), $h$ and $j$ have fixed points 0 and 1 . Now let $r$ be in $[0,1]$ but not in $g^{c} g(r)$. Then $r$ is a local minimum or a local maximum for $g$, so there is an $\varepsilon>0$ such that $g(r)$ is an endpoint of the interval $g([r-\varepsilon, r+\varepsilon])$. There is an integer $n$ such that for all integers $i \geqq n$ and for all $\langle w, x, y, z\rangle$ in $P_{i},|y-z|<\varepsilon$ (since the conditions of the preceding proposition are satisfied). For all $i \geqq n$ and for all $\langle w, x, y, z\rangle$ in $P_{i}$, if $r$ is in $[y, z]$, then $g(r)$ is an endpoint for $g([y, z])$, and therefore, there is at most one element $\langle\bar{w}, \bar{x}, \bar{y}, \bar{z}\rangle$ in the $f-g$ refinement of $\langle w, x, y, z\rangle$ such that $r$ is in the interior of $[\bar{y}, \bar{z}]$. It follows that the chain $P_{n}$ has at least as many elements as the set $h^{c}(r)$; hence $h^{c}(r)$ is a finite set. Similarly, if $s$ is in $[0,1]$ but not in $f^{c} f(s)$, then $j^{c}(s)$ must be a finite set. This completes Step 1 .

Step 2. ( $h$ and $j$ satisfy Step 1, and if $h(x)=h(y)$ and $j(x)=j(y)$ then $\left.h\right|_{[x, y]}$ and $\left.j\right|_{[x, y]}$ are constant.) Let $E$ denote the set of all pairs of functions $\langle h, j\rangle$ such that $h$ and $j$ satisfy the properties of Step 1 . Partially order the set $E$ such that $\langle h, j\rangle$ $\leqq\left\langle h^{\prime}, j^{\prime}\right\rangle$ if and only if for every $x$ in $[0,1]$ either $h(x)=h^{\prime}(x)$ or $h^{\prime}$ is locally constant at $x$ and for every $y$ in $[0,1]$ either $j(y)=j^{\prime}(y)$ or $j^{\prime}$ is locally constant at $y$. Zorn's lemma will now be applied to find a maximal element of $E$. Step 1 shows that $E$ is not empty. If $L=\left\{\left\langle h_{n}, j_{n}\right\rangle / n\right.$ in $\left.N\right\}$ is a nonempty linearly ordered subset of $E$, then define an upper bound $\left\langle h^{*}, j^{*}\right\rangle$ for $L$ in $E$. For each $x$ in $[0,1]$ define $h^{*}(x)=h_{m}(y)$ and $j^{*}(x)=j_{m}(y)$ where $m$ is any element of $N$ and where $y$ is the least upper bound 
of points $z$ with the properties $z<x$ and for all $n$ in $N, h_{n}$ is not locally constant at $z$, provided there is one such $z$; otherwise (i.e. if no such $z$ exists) then let $y$ be the greatest lower bound of points $w$ with the properties $w>x$ and for all $n$ in $N, h_{n}$ is not locally constant at $w$. For every $\left\langle h^{\prime}, j^{\prime}\right\rangle$ in $E$ and each $x$ in $[0,1], h^{\prime}$ is locally constant at $x$ if and only if $j^{\prime}$ is locally constant at $x$, since $f$ and $g$ are nowhere constant and $f j^{\prime}=g h^{\prime}$. Also if $n$ and $m$ are in $N$ with $\left\langle h_{n}, j_{n}\right\rangle \leqq\left\langle h_{m}, j_{m}\right\rangle$ then $h_{n}$ locally constant at some point $x$ implies that $h_{m}$ is locally constant at $x$. Therefore $\left\langle h^{*}, j^{*}\right\rangle$ is an upper bound for $L$ in $E$. So by Zorn's Lemma $E$ has a maximal element; let $\langle h, j\rangle$ be a maximal element of $E$. Assume that $x$ and $y$ are in $[0,1]$ such that $h(x)=h(y)$ and $j(x)=j(y)$. Define $\left\langle h^{\prime \prime}, j^{\prime \prime}\right\rangle$ as follows: $h^{\prime \prime}(z)=h(x)$ and $i^{\prime \prime}(z)=j(x)$ for all $z$ in $[x, y]$; and $h^{\prime \prime}(z)=h(z)$ and $j^{\prime \prime}(z)=j(z)$ for all $z$ in $[0,1]$ but not in $[x, y]$. Now $\left\langle h^{\prime \prime}, j^{\prime \prime}\right\rangle$ is clearly in $E$ and $\langle h, j\rangle \leqq\left\langle h^{\prime \prime}, j^{\prime \prime}\right\rangle$. But $\langle h, j\rangle$ was a maximal element in $E$ so $h=h^{\prime \prime}$ and $j=j^{\prime \prime}$; that is, $\left.h\right|_{[x, y]}$ and $\left.j\right|_{[x, y]}$ are constant. This completes Step 2.

Step 3. ( $h$ and $j$ satisfy the conditions of the lemma.) Let $h^{*}$ and $j^{*}$ satisfy the properties of Step 2. Let $R$ denote the equivalence relation between points defined: $x R y$ if and only if $\left.h^{*}\right|_{[x, y]}$ is constant (or equivalently $\left.j^{*}\right|_{[x, y]}$ is constant). Let $[0,1] / R$ denote the quotient space (defined by identifying points of $[0,1]$ which are equivalent under $R$ with the order topology where the order is inherited from $[0,1])$. Let $p$ be the projection map from $[0,1]$ onto $[0,1] / R$. There is a function $q$ which is monotone with fixed points 0 and 1 such that $\left.q\right|_{[x, y]}$ is constant if and only if $\left.h^{*}\right|_{[x, y]}$ is constant (i.e. if and only if $x R y$ ). Now $q p^{-1}$ (meaning $q\left(p^{-1}(x)\right)$ for each $x$ in $[0,1] / R)$ is a continuous injection mapping $[0,1] / R$ onto $[0,1]$, so $q p^{-1}$ is a homeomorphism. Define $h=h^{*} p^{-1}\left(q p^{-1}\right)^{-1}$ and $j=j^{*} p^{-1}\left(q p^{-1}\right)^{-1}$. Since $h^{*} p^{-1}$ and $j^{*} p^{-1}$ are nowhere constant maps, $h$ and $j$ are functions with fixed points 0 and 1 which are nowhere constant; also $f j=f j^{*} p^{-1}\left(q p^{-1}\right)^{-1}=g h^{*} p^{-1}\left(q p^{-1}\right)^{-1}=g h$. Since $h^{*}$ and $j^{*}$ satisfy the properties of Step 2: if $h(x)=h(y)$ and $j(x)=j(y)$ then $x=y$; if $x$ is not in $g^{c} g(x)$ then $h^{c}(x)$ is a finite set; and if $y$ is not in $f^{c} f(y)$ then $j^{c}(y)$ is a finite set. If $x$ is in $g^{c} g(x)$ then $h^{c}(x) \subset(g h)^{c}(g(x))=(f j)^{c}(g(x))$ (since $g$ is nowhere constant), and hence $j\left(h^{c}(x)\right) \subset j\left((f j)^{c}(g(x))\right) \subset f^{c}(g(x))$ (since $f$ is nowhere constant). Therefore if $x$ is in $g^{c}(g(x))$, then $f^{c}(g(x))$ has at least as many element as $h^{c}(x)$ since it was seen that $\left.j\right|_{h^{-1}(x)}$ is a one-to-one map and hence $j$ sends distinct elements of $h^{c}(x)$ to distinct elements of $f^{c}(g(x))$. Therefore for every $x$ in [0,1], if $f^{c}(g(x))$ is a finite set, then $h^{c}(x)$ is a finite set. Similarly for every $y$ in $[0,1]$, if $g^{c}(f(y))$ is a finite set, then $j^{c}(y)$ is a finite set. This completes Step 3 and the proof of the lemma.

LEMMA 2. Let $f$ and $g$ have fixed points 0 and 1 and suppose $g$ locally constant at $x$ implies that $f^{c}(g(x))$ is a finite set, and $f$ locally constant at $y$ implies that $g^{c}(f(y))$ is a finite set. Then there exist functions $h$ and $j$ with fixed points 0 and 1 such that $f j=g h$.

Proof. Assume that $f$ and $g$ satisfy the hypothesis of the lemma. Let $R$ be the equivalence relation between points in $[0,1]$ defined: $x R y$ if and only if $\left.f\right|_{[x, y]}$ is 
constant. Let $p$ be the projection of $[0,1]$ onto the quotient space $[0,1] / R$, and let $\theta$ be a homeomorphism of $[0,1]$ onto $[0,1] / R$ such that $\theta(0)=p(0)$. Similarly, let $S$ be the eqiuvalence relation between points of $[0,1]$ defined: $x S y$ if and only if $\left.g\right|_{[x, y]}$ is constant. Let $q$ be the projection of $[0,1]$ onto the quotient space $[0,1] / S$, and let $\phi$ be a homeomorphism of $[0,1]$ onto $[0,1] / S$ such that $\phi(0)=q(0)$.

Observe that $f p^{-1}$ and $g q^{-1}$ are well defined (point valued) maps, and that $f p^{-1} \theta$ and $g q^{-1} \phi$ are nowhere constant functions with fixed points 0 and 1 . Let $\bar{h}$ and $j$ be nowhere constant functions (whose existences are assured by the preceding lemma) with fixed points 0 and 1 such that $f p^{-1} \theta j=g q^{-1} \phi \bar{h}$; if $\left(f p^{-1} \theta\right)^{c}\left(g q^{-1} \phi(x)\right)$ is a finite set then $\bar{h}^{c}(x)$ is a finite set; and if $\left(g q^{-1} \phi\right)^{c}\left(f p^{-1} \theta(y)\right)$ is a finite set then $\bar{j}^{c}(y)$ is a finite set.

Let $A$ be the set of points, $x$, such that there exists $y$ with $y \neq x$ and $x R y$; let $B$ be the set of points, $x$, such that there exists $y$ with $y \neq x$ and $x S y$. For each $x$ in $B$, $f^{c}(g(x))$ is a finite set (by the hypothesis), hence $\left(f p^{-1} \theta\right)^{c}(g(x))$ is a finite set, and so $\bar{h}^{c}\left(\phi^{-1} q(x)\right)$ must be a finite set. Similarly, for each $x$ in $A, \bar{j}^{c}\left(\theta^{-1} p(x)\right)$ is a finite set. Now since $p(A)$ and $q(B)$ are countable sets, $\bar{j}^{c}\left(\theta^{-1} p(A)\right)$ and $\bar{h}^{c}\left(\phi^{-1} q(B)\right)$ must also be countable sets (possibly finite or empty). Let $\left\{x_{i} / i=1,2, \ldots\right\}$ be an enumeration of $\bar{j}^{c}\left(\theta^{-1} p(A)\right) \cup \bar{h}^{c}\left(\phi^{-1} q(B)\right)$ such that $x_{i} \neq x_{i^{\prime}}$ whenever $i \neq i^{\prime}$. Let $I$ be the topological space defined by splicing an interval into $[0,1]$ at $x_{i}$ for each $i=1,2, \ldots$; specifically, $I$ is the set $[0,1]$ with $x_{i}$ replaced by $[-1 / 2 i,-1 /(2 i+1)]$ for each $i=1,2, \ldots$, and $I$ is topologized with the « order topology where $\ll$ is a linear order on $I$ defined for any $x$ and $y$ in $I$ as $x \ll y$ provided $x$ and $y$ are both in $[0,1]$ or both in $[-1 / 2 i,-1 /(2 i+1)]$ for some $i=1,2, \ldots$, and $x<y ; x$ is in $[0,1], y$ is in $[-1 / 2 i,-1 /(2 i+1)]$ for some $i=1,2, \ldots$, and $x<x_{i} ; x$ is in $[-1 / 2 i,-1 /(2 i+1)]$ for some $i=1,2, \ldots, y$ is in $[0,1]$, and $x_{i}<y$; and $x$ is in $[-1 / 2 i,-1 /(2 i+1)]$ for some $i=1,2, \ldots, y$ is in $\left[-1 / 2 i^{\prime},-1 /\left(2 i^{\prime}+1\right)\right]$ for some $i^{\prime}=1,2, \ldots$, and $x_{i}<x_{i^{\prime}}$.

Now observe that for each $i=1,2, \ldots$, such that $x_{i} \neq 0$, the limit of $p^{-1} \theta j(x)$ as $x$ approaches $x_{i}$ from below is a well-defined point. If $p^{-1} \theta j\left(x_{i}\right)$ is a singleton, the limit in question equals this $p^{-1} \theta j\left(x_{i}\right)$. If $p^{-1} \theta j\left(x_{i}\right)$ is not a singleton, then it is a closed interval and $f$ restricted to this interval is constant. Hence (by hypothesis) $g^{c}\left(f p^{-1} \theta j\left(x_{i}\right)\right)$ is a finite set, so $\left(g q^{-1} \phi\right)^{c}\left(f p^{-1} \theta j\left(x_{i}\right)\right)$ is a finite set, and so $\bar{j}^{c}\left(j\left(x_{i}\right)\right)$ is a finite set. Therefore there exists a real number $\varepsilon>0$ such that $j\left(x_{i}\right)$ is an endpoint of the interval $j\left(\left[x_{i}-\varepsilon, x_{i}\right]\right)$, and hence the limit of $p^{-1} \theta j(x)$ as $x$ approaches $x_{i}$ from below will be one endpoint of $p^{-1} \theta j\left(x_{i}\right)$. Similarly, for each $i=1,2, \ldots$, the limit of $p^{-1} \theta j(x)$ and the limit of $q^{-1} \phi \bar{h}(x)$ each is a well-defined point as $x$ approaches $x_{i}$ from below (unless $x_{i}=0$ ) and also as $x$ approaches $x_{i}$ from above (unless $x_{i}=1$ ).

Define $j^{\prime}$ mapping $I$ onto $[0,1]$ by the rule $\left.j^{\prime}\right|_{I \cap[0,1]}=\left.p^{-1} \theta j\right|_{I \cap[0,1]}$, and for each $i=1,2, \ldots,\left.j^{\prime}\right|_{[-1 / 2 i,-1 /(2 i+1)]}$ is the unique linear map such that $j^{\prime}(-1 / 2 i)$ is the limit of $p^{-1} \theta j(x)$ as $x$ approaches $x_{i}$ with $0 \leqq x<x_{i}$ (or if $x_{i}=0$, then $j^{\prime}(-1 / 2 i)=0$ ), and $j^{\prime}(-1 /(2 i+1))$ is the limit of $p^{-1} \theta j(x)$ as $x$ approaches $x_{i}$ with $x_{i}<x \leqq 1$ (or if $x_{i}=1$, then $\left.j^{\prime}(-1 /(2 i+1))=1\right)$. Similarly, define $h^{\prime}$ mapping $I$ onto $[0,1]$ by the rule: $\left.h^{\prime}\right|_{I \cap[0,1]}=\left.q^{-1} \phi \bar{h}\right|_{I \cap[0,1]}$, and for each $i=1,2, \ldots,\left.h^{\prime}\right|_{[-1 / 2 i,-1 /(2 i+1)]}$ is the 
unique linear map such that $h^{\prime}(-1 / 2 i)$ is the limit of $q^{-1} \phi \bar{h}(x)$ as $x$ approaches $x_{i}$ with $0 \leqq x<x_{i}$ (or if $x_{i}=0$, then $\left.h^{\prime}(-1 / 2 i)=0\right)$, and $h^{\prime}(-1 /(2 i+1)$ ) is the limit of $q^{-1} \phi \bar{h}(x)$ as $x$ approaches $x_{i}$ with $x_{i}<x \leqq 1$ (or if $x_{i}=1$, then $h^{\prime}(-1 /(2 i+1))=1$ ).

The cluster points in $I$ of $\{(-1 / 2 i) / i=1,2, \ldots\}$ coincide with the cluster points in $I$ of $\{(-1 /(2 i+1)) / i=1,2, \ldots\}$, and $h^{\prime}$ and $j^{\prime}$ are clearly continuous at all points which are not one of these cluster points. Let $y$ be one of these cluster points. If $y$ is approached in $I$ from below by points of the form $-1 / 2 i(i=1,2, \ldots)$ then $y$ is also approached in $I$ from below by points of the form $-1 /(2 i+1)(i=1,2, \ldots)$, and $j^{\prime}(y)$ equals the limit of $j^{\prime}(-1 / 2 i)$ and also the limit of $j^{\prime}(-1 /(2 i+1))$ as $-1 / 2 i$ approaches $y$ from below $(i=1,2, \ldots)$. Therefore $j^{\prime}$ is continuous from below, and similarly $j^{\prime}$ is continuous from above. Hence $j^{\prime}$ is continuous; similarly, $h^{\prime}$ is continuous.

For each $x$ in $I \cap[0,1], f j^{\prime}(x)=f p^{-1} \theta j(x)=g q^{-1} \phi \bar{h}(x)=g h^{\prime}(x)$. Also, since $f$ and $g$ are continuous, $f j^{\prime}(-1 / n)=g h^{\prime}(-1 / n)$ for $n=2,3, \ldots$, so for each $i=1,2, \ldots$, the linear functions $\left.f j^{\prime}\right|_{[-1 / 2 i,-1 /(2 i+1)]}$ and $\left.g h^{\prime}\right|_{[-1 / 2 i,-1 /(2 i+1)]}$ coincide. Therefore $f j^{\prime}=g h^{\prime}$.

Let $\psi$ be a homeomorphism of $[0,1]$ onto $I$ such that $\psi(0)=0$ if 0 is in $I$, or $\psi(0)=-1 / 2 i$ where $x_{i}=0$ otherwise. Let $h=h^{\prime} \psi$ and $j=j^{\prime} \psi$. The two functions $h$ and $j$ have fixed points 0 and 1 , and $f j=g h$. The proof of the lemma is complete.

Definition 6. When $f([a, b])=g([c, d])$, the pair of points $\langle w, y\rangle$ will be called $\left.f\right|_{[a, b]}-\left.g\right|_{[c, d]}$ consistent provided there exists a sequence $\left\{\left\langle w_{i}, y_{i}\right\rangle / i=0,1, \ldots\right\}$ in the product $[a, b] \times[c, d]$ converging to $\langle w, y\rangle$ such that $f\left(w_{0}\right)=g\left(y_{0}\right), f\left(w_{0}\right)$ is an endpoint of the interval $f([a, b])$, and for each $i=1,2, \ldots$ the four intervals $f\left(\left[w_{i}, w_{i-1}\right]\right), g\left(\left[y_{i}, y_{i-1}\right]\right),\left[f\left(w_{i}\right), f\left(w_{i-1}\right)\right]$, and $\left[g\left(y_{i}\right), g\left(y_{i-1}\right)\right]$ coincide.

Proposition 2. If $\langle a, c\rangle$ and $\langle b, d\rangle$ are $f-g$ consistent, if $g$ locally constant at $x$ implies that $f^{c}(g(x))$ is a finite set, and if $f$ locally constant at $y$ implies that $g^{c}(f(y))$ is a finite set, then there exist functions $h$ and $j$ such that $f j=g h, j(0)=a, j(1)=b$, $h(0)=c$, and $h(1)=d$.

Proof. There exist points $r, s, u$, and $v$ in $[0,1]$ such that $\langle r, 0\rangle,\langle s, 1\rangle,\langle 0, u\rangle$, and $\langle 1, v\rangle$ are $f-g$ consistent. By the preceding lemma, if $w, x, y$, and $z$ are in $[0,1]$ such that $f(w)=g(y)$ and the four intervals $f([w, x]),[f(w), f(x)], g([y, z])$, and $[g(y), g(z)]$ coincide, and if $t$ and $t^{\prime}$ are distinct points, then there exist continuous maps $h^{\prime}$ and $j^{\prime}$ with $h^{\prime}$ mapping $\left[t, t^{\prime}\right]$ onto $[y, z]$ and $j^{\prime}$ mapping $\left[t, t^{\prime}\right]$ onto $[w, x]$ such that $j^{\prime}(t)=w, j^{\prime}\left(t^{\prime}\right)=x, h^{\prime}(t)=y, h^{\prime}\left(t^{\prime}\right)=z$, and $f j^{\prime}=g h^{\prime}$. Hence the desired functions $h$ and $j$ can be constructed by piecing together appropriate maps defined on subintervals of $[0,1]$ by the preceding lemma.

COROLLARY 1. If each of $f$ and $g$ is either a nowhere constant function or has the property that the inverse of each point in $[0,1]$ is a set with a finite number of components, then there exist functions $h$ and $j$ such that $f j=g h$. 
COROLlaRY 2. For any functions $f$ and $g$ and for any $\varepsilon>0$, there exist functions $h$ and $j$ such that $\|f j-g h\|<\varepsilon$ (i.e. for every $x$ in $[0,1],|f j(x)-g h(x)|<\varepsilon)$.

Proof. By the preceding corollary, it is sufficient to define nowhere constant functions $f^{\prime}$ and $g^{\prime}$ such that $\left|f^{\prime}-f\right|<\varepsilon / 2$ and $\left|g^{\prime}-g\right|<\varepsilon / 2$.

COROLlaRY 3. There exist functions $h$ and $j$ such that $f j=g h$ if and only if there exists a linearly ordered set of $f-g$ weakly compatible chains $\left\{P_{i} / i=0,1, \ldots\right\}$ where for each $i=0,1, \ldots$

$$
P_{i}=\left\{\left\langle w_{i^{\prime}}^{i}, x_{i^{i}}^{i}, y_{i^{\prime}}^{i}, z_{i^{\prime}}^{i}\right\rangle / i^{\prime}=1, \ldots, n_{i}\right\}
$$

and for each $i^{\prime}=1, \ldots, n_{i}$ if $\left|w_{i^{\prime}}^{i}-x_{i^{\prime}}^{i}\right|+\left|y_{i^{\prime}}^{i}-z_{i^{\prime}}^{i}\right| \geqq 1 / i$ then $\left.g\right|_{\left[y_{i}^{i}, z_{i}^{i},\right]}$ locally constant at $r$ implies that $\left.f\right|_{\left[w_{i}^{i}, x_{i}^{i},\right]} ^{c}(g(r))$ is a finite set; $\left.f\right|_{\left[w_{i}^{i}, x_{i}^{i},\right]}$ locally constant at $s$ implies

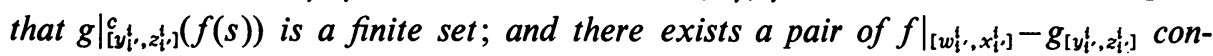
sistent points in

$$
\left(\left[w_{i^{i}}^{i}, x_{i^{\prime}}^{i}\right] \cap\left[w_{i^{\prime \prime}}^{i}, x_{i^{\prime \prime}}^{i}\right]\right) \times\left(\left[y_{i^{\prime}}^{i}, z_{i^{\prime}}^{i}\right] \cap\left[y_{i^{\prime \prime}}^{i}, z_{i^{\prime \prime}}^{i}\right]\right)
$$

for $i^{\prime \prime}=i^{\prime}-1$ (unless $\left.i^{\prime}=1\right)$ and for $i^{\prime \prime}=i^{\prime}+1$ (unless $\left.i^{\prime}=n_{i}\right)$.

\section{REFERENCES}

1. J. P. Huneke, On common fixed points of commuting continuous functions on an interval, Trans. Amer. Math. Soc. 139 (1969), 371-381.

2. J. V. Whittaker, A mountain-climbing problem, Canad. J. Math. 18 (1966),873-882.

\section{UNIVERSITY OF MINNESOTA,}

MinNEAPOLIS, MinNESOTA 